International Journal of Wireless \& Mobile Networks (IJWMN) Vol. 3, No. 5, October 2011

\title{
ChanNel ASSignMent AlgorithMS FOR MRMC WIRELESS MESH NETWORKS
}

\author{
Mohammad A Hoque ${ }^{1}$ and Xiaoyan Hong ${ }^{2}$ \\ Department of Computer Science, The University of Alabama, USA \\ ${ }^{1}$ mhoque@cs.ua.edu, ${ }^{2}$ hxy@cs.ua.edu
}

\begin{abstract}
The wireless mesh networksare considered as one of the vital elements in today's converged networks,providing high bandwidth and connectivity over large geographical areas. Mesh routers equipped with multiple radios can significantly overcome the capacity problem and increase the aggregate throughput of the network where single radio nodessuffer from performancedegradation. Moreover, the market availability of cheap radios or network interfaces also makes multi-radio solutions more feasible.A key issue in such networks is how to efficiently design a channel assignment scheme that utilizes the available channels as well as increases overall performance of the network. This paper provides an overall review on the issues pertaining to the channel assignment in WMNs and the most relevant approaches and solutions developed in the area. They include design challenges, goals and criteria; routing considerations, graph based solutions and challenges of partially overlapped channels. We conclude that the assignment of channels to the radio interfaces continuously poses significant challenges. Many research issues remain open for further investigation.
\end{abstract}

\section{KEYWORDS}

Channel assignment, wireless multi-hop routing, multiple radios and multiple channels, wireless mesh networks, partially overlapping channels.

\section{INTRODUCTION}

Wireless Mesh Network (WMN) provides a very reliable and cost efficient alternative for Internet connectivityover wide areasand enables ubiquitous computing environment through multi-hop relay[1]. In real world implementation of $\mathrm{WMN}$, the total number of network interfacesis muchgreater than the number of frequency channels available for transmission. Moreover, each wireless node can have more than one interfaces or radios. This may lead to a topology where many mutually interfering links are assigned to the set of channels. This interference between concurrent transmissions can detrimentally degrade the throughput or performance of these networks. Therefore, as with cellular networks, the key factor for minimizing the effect of interference is the efficient reuse of radio frequency. One of the major issues concerned with WMN architecture supporting multiple radios and multiple channels (MRMC)is the channel assignment (CA) problem. Particularly for multi-hop networks, it is very complex to design an optimized CA algorithm that makes efficient utilization of available channels and at the same time minimizes the overall network interferences. In general, channel assignment algorithms shouldfacilitate multi-path routing amongwireless routers apart from minimizing interference onany given channel or from adjacent channels.

Existing channel assignment algorithms designedfor multi-radio multi-channel wireless mesh networks(MRMC-WMN) mostly deal with orthogonal or non-overlappingchannels. Recently 
the limited availabilityof orthogonal channelsin dense networks has motivated the wireless research community to studypartially overlapped channels (POC), which are consideredas a great potential for increasing the number ofsimultaneous transmissions and eventually upgrading thenetwork capacity; especially in the case of MRMC-WMN.

A substantial amount of research has been done so farwith multi-dimensional categories of channel assignmentschemes in wireless networks $[2,3,9,12-20,39,40]$. In this paper, variousaspects of channel assignment algorithm are discussedfrom different perspectives. Specifically, we outline objectives,design features that differ one from other solutionsand set them into different categories. We thendiscuss in details about a few representative solutions.Our emphasis then goes on graph based approaches andsolutions using POC. In all, our scope of this papergradually narrows down starting from the broad area ofchannel assignment (CA) to the depth of using POCs.

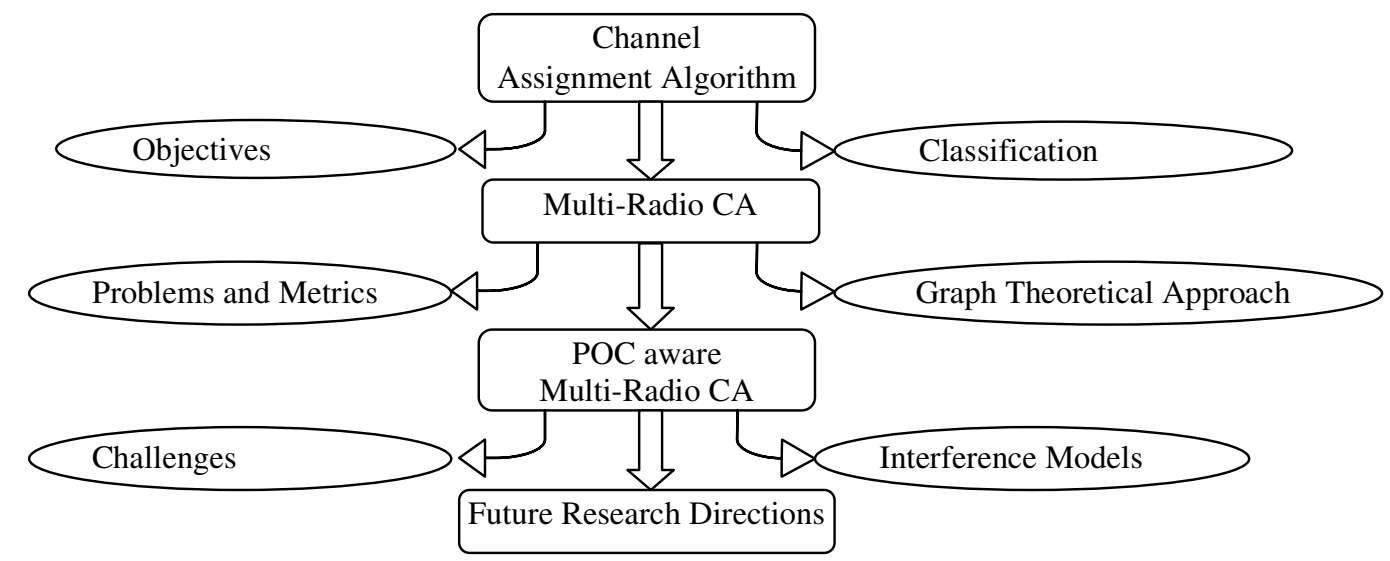

Figure 1.Organization of Channel Assignment Survey

The organization of the paper is illustrated in Figure1. The paper has three main parts. In the first part, consistingof Sections II and III, we discuss research issuesof channel assignment algorithms in general. In SectionII, we begin with outlining the common objectives inthe existing CA algorithms, and in Section III, we depictvarious ways that the classifications of the CA algorithmshave been used by many researchers. After that, in thesecond part, which consist of Sections IV, V and VI, weconcentrate on issues particularly related to the multipleradio environments. In section IV we argue in favor ofthe deployment of multi-radio communications followedby the choice of routing protocols associated with suchdeployment in section V. Later we present a graphtheoretical framework of formulating channel assignmentproblems in section VI. Then, in the third part, PartiallyOverlapped Channel (POC) related design issues areenumerated in section VII. Finally, we discuss the futureresearch directions in Section VIII and conclude thepaper in Section IX.

\section{Characteristicsof Efficient Channel Assignment Algorithm}

In the literature, solving channel assignment problems have been targeted to meet various design objectives. Some of these goals are described below.

One of the key objectives that need to be considered while designing a channel assignment scheme is to minimize the network interference. This interference minimization goal can either be implemented globally (in case of centralized schemes) or locally (in case of distributed schemes). It has been proved in the literature that the channel interference effects 
can cause a significant throughput loss in the network, especially if the design includes partially overlapped channels. Hence, most of the channel assignment algorithms should focus on this issue with severe importance.

All wireless networks are subject to capacity limitations due to many issues related to the characteristics of physical media. So a common goal for any wireless design is to focus on increasing capacity by applying innovative channel assignment schemes that canmaximize the overall network throughput. Throughput is often regarded as the primary criterion to evaluate the efficiency of a new scheme. In fact, throughput is maximized by increasing the number of parallel transmission in a network. So, channel assignment algorithms should equally focus on throughput maximizing.

The IEEE 802.11 standard specifies multiple non-overlapping channels for use (3 in $802.11 \mathrm{~b} / \mathrm{g}, 12$ in 802.11a). So the channel assignment scheme should aim into exploiting channel diversity to maximize spectrum utilization. Also, carefully allocating partially overlapped channels with proper interference model can further improve the channel utilization to maximum level. Therefore, researchers of wireless mesh networks are emphasizing on increasing channel diversity while designing channel allocation schemes.

Adaptation to changing traffic conditions is another important criterion for a well designed channel assignment scheme. An efficient channel assignment algorithm should not only maximize channel utilization but also distribute the load equally among different channels.

Inefficient channel assignment may lead to network partitions which ultimately deforms the original topology. So, preserving the topology by avoiding network partition is also an important goal for channel assignment algorithms.

\section{Classification Of Channel Assignment Schemes}

The channel assignment schemes can be classified based on different criteria and perspectives. Table I summarizes the classification followed by the description of each category thereafter. It is noteworthy that, these categories are not necessarily disjoint from each other. A particular type of scheme based on one criterion may fully or partially overlap with another type in different criteria.

Table 1.Classification of Channel Assignment Algorithms

\begin{tabular}{|l|l|}
\hline Classification Criteria & Types of Channel Assignment \\
\hline Channel Switching Frequency & a) Static/Fixed: \\
& $\begin{array}{l}\text { - Common Channel Assignment (CCA) } \\
\text { - Varying Channel Assignment (VCA) }\end{array}$ \\
& b) Dynamic \\
& c) Hybrid \\
\hline Number of Radios & a) Single Radio \\
& b) Multiple Radio \\
\hline Spectrum Utilization & a) Orthogonal Channels (OCs) \\
& b) Partially Overlapped Channels (POCs) \\
\hline Topology Awareness & a) Centralized \\
& b) Distributed \\
\hline Routing Dependency & a) Routing independent \\
& b) Routing dependent \\
& c) Joint Approach \\
\hline
\end{tabular}


International Journal of Wireless \& Mobile Networks (IJWMN) Vol. 3, No. 5, October 2011

\begin{tabular}{|l|l|}
\hline Infrastructure & a) Access Point based \\
& b) Ad hoc based \\
& c) Hybrid approach \\
\hline Granularity of Assignment & a) Per Packet Channel Assignment \\
& b) Per link Channel Assignment \\
& c) Per Flow Channel Assignment \\
& d) Per Component Channel Assignment \\
\hline
\end{tabular}

\subsection{Based on Channel Switching Frequency}

Skalliet. al. [40] proposed a taxonomical classification of various channel assignment schemes based on the criteria of channel switching frequency where the channel assignment schemes are divided into three main categories: fixed, dynamic and hybrid.

\subsubsection{Fixed/Static Channel Assignment}

Fixed or Static assignment schemes assign each radio to a channel for a relatively long period of time. The purpose of fixed channel assignment is to control the connectivity of the nodes.Das et. al [53] described some of the key issues related to static channel assignment algorithms. Fixed channel assignmentscheme has been further subcategorized into two types:

Common Channel Assignment (CCA) is the simplest among all the schemes where the network interfaces of each node are assigned to a common set of channels. The primaryadvantage of this approach is that the network topology essentially remains identical tothatusing a single channel assignment scheme, while increasingthe network throughput by the use of multiple channels. However, in case where the number of orthogonal channels is greater than the number of radios in each node, the throughput gain may be limited andmay lead to inefficient channel utilization.

In case of Varying Channel Assignment (VCA), radios of different nodes are assigned to different sets of channels. However, assigning disjoint set of channels to the NICs may lead to network isolation and modified topology.An example of this type of algorithms is Connected Low Interference Channel Assignment (CLICA) [41].

\subsubsection{Dynamic Channel Assignment}

In Dynamic assignment schemes, any radiocan be assigned to any channel where theradios can frequently switch from one channel to another. The advantage of dynamic assignment is that it utilizes multiple channels with few interfaces. However, these approaches have the disadvantage of strict time synchronization requirement between the nodes. Other key challengesconstitute of channel switching delays and the need for signalling and coordination mechanisms for channel switching between a pair of nodes. These constraints impose practical challenges forimplementation in real networks.

\subsubsection{Hybrid Channel Assignment}

Hybrid channel assignment strategies combine both fixed and dynamic assignment strategies. Here, some radiosare assigned to a static channel whereas others can be dynamically switched between several channels.

\subsection{Based on Number of Radios}

When all the nodes in a WMN are equipped with single radio, these channel assignment schemes are applicable. Advantages of this type are: (i) no complicacy of self-interference, (ii) channel selection algorithm is quite simple as only one channel has to be selected and finally 
(iii) easy to implement. However, it also has drawbacks like: (i) less channel utilization (ii) no simultaneous transmission possible from a single node (iii) frequent channel switching

Currently thechannel assignment algorithms are targeted for meshnetworks with multi-radio environment. As multiplechannels are utilized at a time, channel utilization ismuch higher. Advantages of multi-radio scheme include(i) less channel switching and (ii) parallel transmissions.However, the channel selection algorithm is complex andinterference handling is also more difficult.

\subsection{Based on SpectrumUtilization}

Currently almost all channelassignment algorithms are designed with nonoverlappingchannels or Orthogonal channels. This doesnot utilize all the available channel resources allocatedfor the specific IEEE 802 technology. For example,in case of IEEE 802.11 $\mathrm{b} / \mathrm{g} / \mathrm{n}$, there are only 3 non-overlappingchannels out of 11 channels (in USA). Duringthe network overload period, there are not sufficientspectrum resources available when using only orthogonalchannels. This initiates the necessity of designing efficientschemes that can utilize all the available channelsin the spectrum.

Recently, a substantial amount of research is goingon with designing channel assignment algorithms with Partially Overlapped Channels (POC). Some of the researchers alreadycame up with efficient algorithms that could handle theinterfering channels. But still questions exist about thefeasibility of implementing those schemes into currentindustry standard. We shall discuss the issues concerningthe POCs later.

\subsection{Based on Topology Awareness}

Centralizedchannel assignment algorithms have the global knowledgeabout the topology, either through global positioningsystem or though routing table information. Theyare mostly useful in case of infrastructure based wirelessnetworks like AP based networks. Centralized algorithmsare easy to implement, less overhead required for routingand node connectivity is determined by access points(APs). In other case, centralized channel assignment isalso applicable without APs when all the nodes have theglobal topology information. In most cases, centralizedalgorithms are either static or quasi-static.

Distributedchannel assignments are the ideal requirement for Adhoc networks. The distributed approach is more feasiblein realistic environments where the global informationfor centralized algorithm is not available. Our previous work [55] summarized a classification of MRMC channel assignment and routing algorithms on the basis of centralized and distributed categories.

\subsection{Based on Routing Dependency}

Most of the channel assignmentschemes are independent of routing protocol.These schemes work with any type of routing protocol,irrespective of proactive or reactive routing categories.Some channel assignmentschemes depend on the type of routing protocol. Thesealgorithms only work with the associated routing protocols.A recent trend is to design jointrouting and channel assignment schemes that optimizethe route by selecting the channels along the end to endpath. In such cases, channel information is also appendedin the routing table and broadcasted periodically. Inthese cross layer designs, efficient routing metric hasto be selected incorporating the channel interferencecharacteristics. An example of such joint approach is theKN-CA algorithm, by Xiaoguang Li et. Al. [24], whichis an enhancement of AODV protocol. 
International Journal of Wireless \& Mobile Networks (IJWMN) Vol. 3, No. 5, October 2011

\subsection{Based on Infrastructure}

Channel assignment schemes that are particularly dependant on infrastructure or based on access point are mostly centralized.In that case the access point has the information of allthe nodes and their adjacent channels. In such case,the access point allocates the channel in a manner thatminimizes the overall interference and maximizes thethroughput and capacity.

On the other hand, ad hoc mesh networkslacks the information of global topology. Henceit is difficult to implement a centralized scheme with thelimited local information. Such centralized design basicallyimposes static channel assignment. Again, usingdistributed approach, the algorithm is prone to inaccuratetopological information which results into networkpartitioning.

In hybridmesh networks, nodes are connected in two ways, one isthe direct single hop connectivity with access point, andanother way is to route through other nodes to connectto a relatively less traffic loaded access point.This type of schemes is applied to areas where load density is high.

\subsection{Based on Granularity}

Per-packetchannel assignment requires more run-time control overheadfor scheduling each single packet with particularchannel. Hence, algorithms in this type are less efficientfor high loads. In [2], [6], Vaidyaet. al., described such a CA scheme where the radios switch from one channel to another in a small time scale. In reality, this type of scheme is not feasible for implementation because of the high overhead.

Inlink-based channel assignmentscheme, channelis assigned to a link between a pair ofnodes, and all packets transmitted between these two nodes usethat particular channel for a certain period of time. Some of the algorithms of this type,focus on assigning channels by ensuringappropriate amount of bandwidth for each linkaccording to the expectedload. On the other hand, other schemesemphasize onminimizing link interference in the network. Several optimization models are also proposedin the literature for centralized channel assignment instatic WMNs, focusing on either maximizing the number simultaneously active links [56] or minimizing the overallinterferences among links.

In flow-based channel assignment scheme, a single channelis assigned to consecutive links along path from source to destination which defines a flow. As for example, So et al.[19]described a channel assignment schemethat binds separate channels to each of the flows ina single radio multichannel network.

Flowbased scheme is extended by Sivakumaret. al. in [20]to component-based channel assignment. A componentis formed by intersecting flows at a particular node and according to this approach an entire componentisassigned a single channel.

\section{Problems With Multi-Radio Channel Assignment}

The IEEE $802.11 \mathrm{~b} / \mathrm{g} / \mathrm{n}$ standards provide 3 and 12 non-overlappingchannels that can be usedin parallel within a mesh network. If multiple radios can be installed on the same node to facilitate the simultaneous use of some of the channels, one can expect increased working bandwidth. Themarket availability of cheap NIC hardwarehas made the multi-radio solutions more feasible.Several research works $[12,13,14]$ have proved that equipping a node with only 2 radios may increase the network capacity as well as throughput by a factor of 6 or 7 . However, beside these benefits, there are a lot of problems associated withmulti-radio channel assignment. Throughout the followingsubsections, we address some of the critical problemsrelated to MRMC design. 
International Journal of Wireless \& Mobile Networks (IJWMN) Vol. 3, No. 5, October 2011

\subsection{Interference Minimization}

Although multi-radio wireless nodes cansignificantly uplift the performance of WMN,there is a critical trade-off to be made between maximizingconnectivity and minimizing interference. Thekey factors to consider are the co-channeland adjacent channel interference due tothe close proximity of the radios equipped on a single node, and those due to the transmissions from neighbouring nodes [35]. The co-channel interference prohibits a particular channel to be used more than once by two links within the interference range simultaneously. The adjacent channel interference determines the total number of usable channels within the neighbourhood (defined by the transmission range). In order to minimize thenetwork interference, a suitable interference model hasto be designed in accordance with the assignable channelsuper set. For example, an interference model which iscapable of handling the self interferenceproblem maynot be suitable for POC based design.

\subsection{Channel Switching Delay}

One of the key challenges in multi-radio environmentinvolve channel switching delay which is typically inthe order of several milliseconds. This mandates tight coordination mechanisms forchannel switching between nodes. Hence, the frequencyof channel switching greatly impacts the efficiency andthroughput of the network.

\subsection{Interdependency with Routing Protocol}

As a matter of fact, routing and channel assignment are interdependent. A routingprotocol selects a path from the source to the destination,and forwards traffic to each link along the path,while channel assignment determines the individual channel that each linkshould use. In other words, CA determines the connectivitybetween two nodes as two radios can only communicatewhen they are tuned to a common channel.Hence channel assignmentultimately determines the network topology. Again, as weknow, routing decisions are dependent on the networktopology which implies thatchannel assignment has a direct impact on routing.Experiments have shown that, dynamically adjusting the channelaccording to thetraffic status can achieve better result, which again proves that routing and channel assignment are tightly coupled.

\subsection{Issues with Joint Channel Assignment and Routing}

In order to maximize the performance gain in MRMC-WMN, joint implementation of routing and channel assignment is very important. Traditional wireless routing protocols $[7,8,11]$ may not provide optimized performance without incorporating integration with CA. Wireless researchers focussing on cross layer protocol design mostly deal with integrating routing with CA. Some of these schemes are designed as centralised algorithm [14, 24, 27, 41] while others considered distributed mode $[9,37]$. However, there are several challengesin effectively designing algorithms for joint $\mathrm{CA}$ androuting, especially in a distributed fashion. More complicacyarises when the network is a heterogeneous typeof multi-radio wireless networks. Below we mentionsome of the critical issues while designing a joint CAand routing algorithm:

For any routing protocol whether or not integrated with CA, arouting metric needsto be concretely defined as a quantitative measurement of the performance gain.In case of joint CA and routing, most of these metrics are defined as compound metric derived from other elementary routing metrics. One such algorithm ofthis type [37] defines a metric named Channel Cost Metric(CCM) thatcomputes the expected transmission costweighted by channel utilization.CCM quantifies the effect of channel interferences along withthe benefit of channel diversity. 
Another major issue arises in networks with heterogeneous radiosoperating with different transmission power and frequency.It can be possible thatthere be no common radio or commonchannel supported in the whole network for both datatransmission and signalling (e.g., routing message), leading to network partitioning.Bhandari and Vaidya [38,42] revealed many issues particularly applicable for networks with heterogeneous radios. Further, reducing the protocol overhead for a distributed algorithm in such a heterogeneous wirelessenvironment presents significant challenges for thejoint implementationof $\mathrm{CA}$ and routing.

\section{Choice Of Routing Metric Integrated With CA}

\subsection{Evolution of Routing Metrics}

In this section, we discuss the routing metrics that havebeen widely accepted for mesh networks in a hierarchical representationbased on their derivation. Some of the well knownrouting metrics are: hop count, RTT, ETX[4], ETT[5], WCETT[5],EDR [10], CCM[37], MCR[15], MIC[18], ILA[48] and iAWARE[50]. Addagaddaet. al. [47] summarized some of the notable features of these routing metrics and proposed modifications over ILA and iAWARE. All these metrics aretopology-dependent and mostmetricswereproposed as improvement over some other previous metrics. Figure 2shows a hierarchical representation of the metrics based on their derivation.

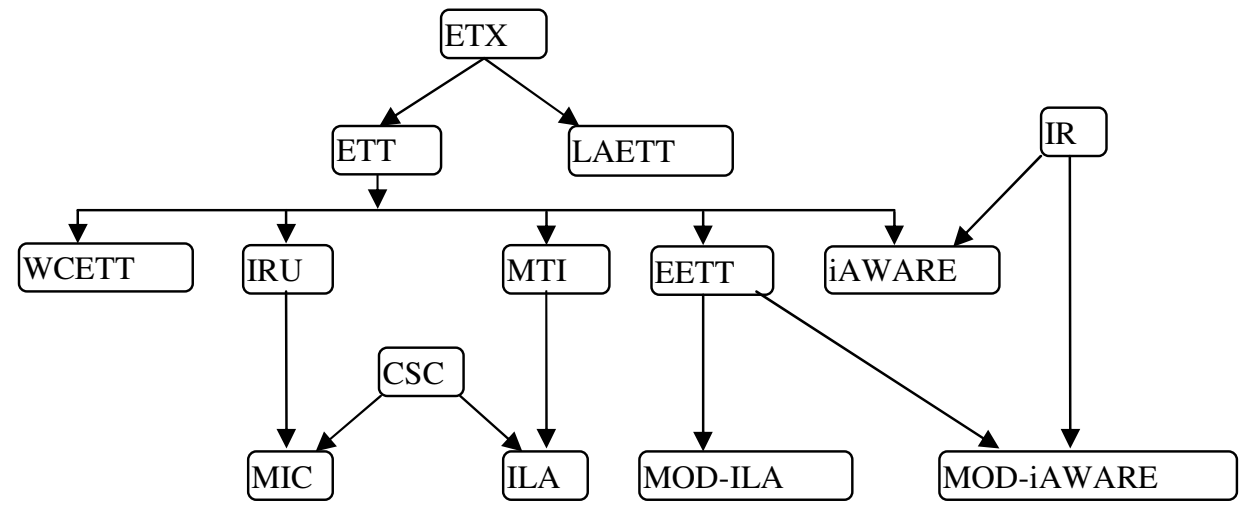

Figure 2. The hierarchical representation of the metric

We also tabulated some of the interesting characteristicsof these metrics under Table 2. These characteristicsgave us a foundation to classify the metrics from twodifferent perspectives, i.e. we categorized the routingmetrics based on isotonicity and also based on interferenceconsideration. 
International Journal of Wireless \& Mobile Networks (IJWMN) Vol. 3, No. 5, October 2011

Table 2.Summary of characteristics of the routing metrics used in wireless networks

\begin{tabular}{|c|c|c|c|c|c|c|c|c|c|c|c|c|}
\hline Characteristics & $\stackrel{0}{\underline{\theta}}$ & $\vec{x}$ & 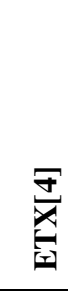 & $\underset{n}{n}$ & $\begin{array}{l}n \\
0 \\
0 \\
0 \\
0\end{array}$ & $\sum_{\bigcup}^{\sqrt{n}}$ & 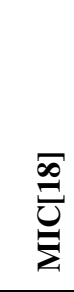 & 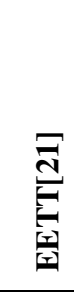 & 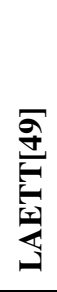 & $\underset{⿱ ㇒}{\stackrel{\infty}{*}}$ & $\frac{\overline{8}}{\sqrt[0]{0}}$ & 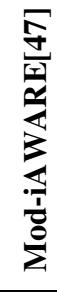 \\
\hline Multi-channel Support & $\mathrm{X}$ & $\mathrm{X}$ & $\mathrm{X}$ & $\mathrm{X}$ & $\mathrm{Y}$ & $\mathrm{Y}$ & $\mathrm{Y}$ & $\mathrm{Y}$ & $\mathrm{Y}$ & $\mathrm{Y}$ & $\mathrm{Y}$ & $\mathrm{Y}$ \\
\hline Intra-Flow Interference & $\mathrm{X}$ & $X$ & $\mathrm{Y}$ & $\mathrm{X}$ & $\mathrm{Y}$ & $\mathrm{Y}$ & $\mathrm{Y}$ & $\mathrm{Y}$ & $\mathrm{X}$ & $\mathrm{Y}$ & $\mathrm{Y}$ & $\mathrm{Y}$ \\
\hline Inter-flow Interference & $X$ & $X$ & $X$ & $\mathrm{X}$ & $\mathrm{X}$ & $\mathrm{Y}$ & $\mathrm{Y}$ & $\mathrm{Y}$ & $\mathrm{X}$ & $\mathrm{Y}$ & $\mathrm{Y}$ & $\mathrm{Y}$ \\
\hline Load balancing & $\mathrm{X}$ & $\mathrm{Y}$ & $\mathrm{X}$ & $\mathrm{X}$ & $\mathrm{X}$ & $\mathrm{Y}$ & $\mathrm{X}$ & $\mathrm{X}$ & $\mathrm{Y}$ & $\mathrm{X}$ & $\mathrm{X}$ & $\mathrm{Y}$ \\
\hline Link loss ratio & $\mathrm{X}$ & $\mathrm{X}$ & $\mathrm{Y}$ & $\mathrm{Y}$ & $\mathrm{Y}$ & $\mathrm{Y}$ & $\mathrm{Y}$ & $\mathrm{Y}$ & $\mathrm{Y}$ & $\mathrm{X}$ & $\mathrm{Y}$ & $\mathrm{Y}$ \\
\hline Throughput & $\mathrm{X}$ & $\mathrm{X}$ & $\mathrm{Y}$ & $\mathrm{Y}$ & $\mathrm{Y}$ & $\mathrm{Y}$ & $\mathrm{Y}$ & $\mathrm{Y}$ & $\mathrm{Y}$ & $\mathrm{Y}$ & $\mathrm{Y}$ & $\mathrm{Y}$ \\
\hline Transmission Rate & $\mathrm{X}$ & $\mathrm{X}$ & $\mathrm{X}$ & $\mathrm{Y}$ & $\mathrm{Y}$ & $\mathrm{Y}$ & $\mathrm{Y}$ & $\mathrm{Y}$ & $\mathrm{Y}$ & $\mathrm{Y}$ & $\mathrm{Y}$ & $\mathrm{Y}$ \\
\hline Link Capacity & $\mathrm{X}$ & $X$ & $\mathrm{X}$ & $\mathrm{Y}$ & $\mathrm{Y}$ & $\mathrm{Y}$ & $\mathrm{Y}$ & $\mathrm{Y}$ & $\mathrm{Y}$ & $\mathrm{Y}$ & $\mathrm{Y}$ & $\mathrm{Y}$ \\
\hline Multi-Radio Support & $\mathrm{X}$ & $\mathrm{X}$ & $\mathrm{Y}$ & $\mathrm{Y}$ & $\mathrm{Y}$ & $\mathrm{Y}$ & $\mathrm{Y}$ & $\mathrm{Y}$ & $\mathrm{Y}$ & $\mathrm{Y}$ & $\mathrm{Y}$ & $\mathrm{Y}$ \\
\hline Heterogenous Radio & $X$ & $\mathrm{X}$ & $\mathrm{X}$ & $\mathrm{X}$ & $\mathrm{X}$ & $\mathrm{Y}$ & $\mathrm{X}$ & $\mathrm{X}$ & $\mathrm{X}$ & $\mathrm{X}$ & $\mathrm{X}$ & $\mathrm{X}$ \\
\hline Agility & $\mathrm{Y}$ & $\mathrm{Y}$ & $\mathrm{X}$ & $\mathrm{X}$ & $\mathrm{X}$ & $\mathrm{X}$ & $\mathrm{X}$ & $\mathrm{X}$ & $\mathrm{X}$ & $\mathrm{X}$ & $\mathrm{X}$ & $\mathrm{X}$ \\
\hline Isotonicity & $\mathrm{Y}$ & $\mathrm{Y}$ & $\mathrm{Y}$ & $\mathrm{Y}$ & $\mathrm{X}$ & $\mathrm{X}$ & $\mathrm{Y}$ & $\mathrm{Y}$ & $\mathrm{Y}$ & $\mathrm{X}$ & $\mathrm{X}$ & $\mathrm{Y}$ \\
\hline
\end{tabular}

\subsection{Classification Based on Isotonicity}

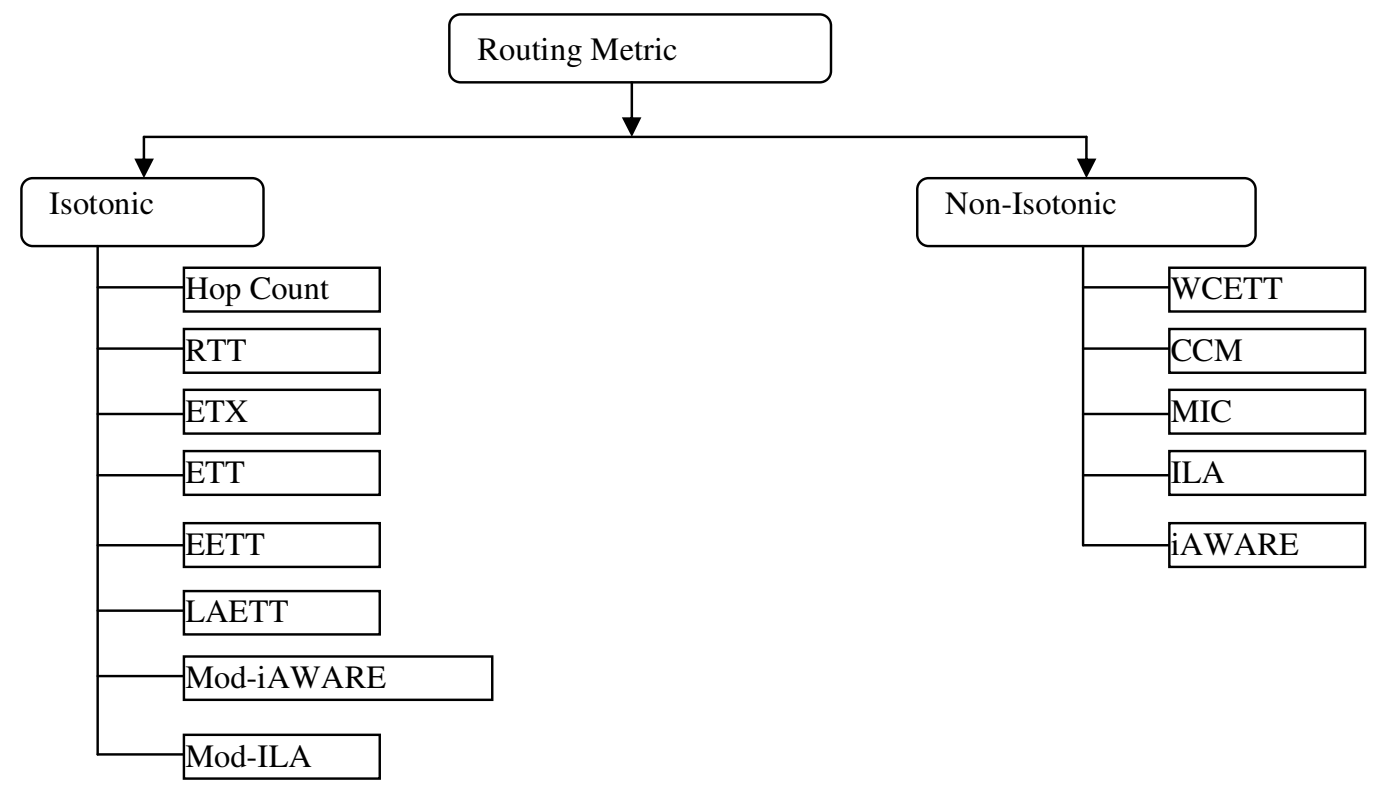

Figure 3. Classification based on Isotonicity

In order to calculate the minimum cost path, most routing protocols follow certain variations of efficient algorithms, like Bellman- Ford or Dijkstra's algorithms. Even if a metric guarantees that its minimum costroute has good performance, there is no assurance ofhaving an efficient algorithm to compute the path cost based on the metric. The property that ensures the existence of such efficient algorithmis called isotonicity [45]. Based on this property, 
routing metrics can broadly be categorized into two classes, namely i) Isotonic and ii) NonIsotonic. Figure 3 shows the classification of some of the common routing metrics on the basis of isotonicity.

\subsection{Classification Based on Interference}

While designing a routing metric, two types of interferences are needed to be considered in a mesh network:

Intra-flow interference occurs while the network interfaces of two or more consecutive links belonging to a single path or flow operate on the same channel.This type of interferences can be mitigated by applying channel diversity; for example, by selecting non-overlapping or orthogonal channels for subsequentlinks. Typically the interference range isgreater than transmission range beyond immediate neighbors. This might result into interference among non-adjacent links operating on same channel in a multi-hop path.

Inter-flow interference is caused by interference generated from other flows that are operating on the same channels. Due to the involvement of multiple flows and routes, controlling interflow interference is more complicated than intra-flow interference. Based on the consideration of these interferences, routing metrics can be classified to four categories as shown in Figure 4.

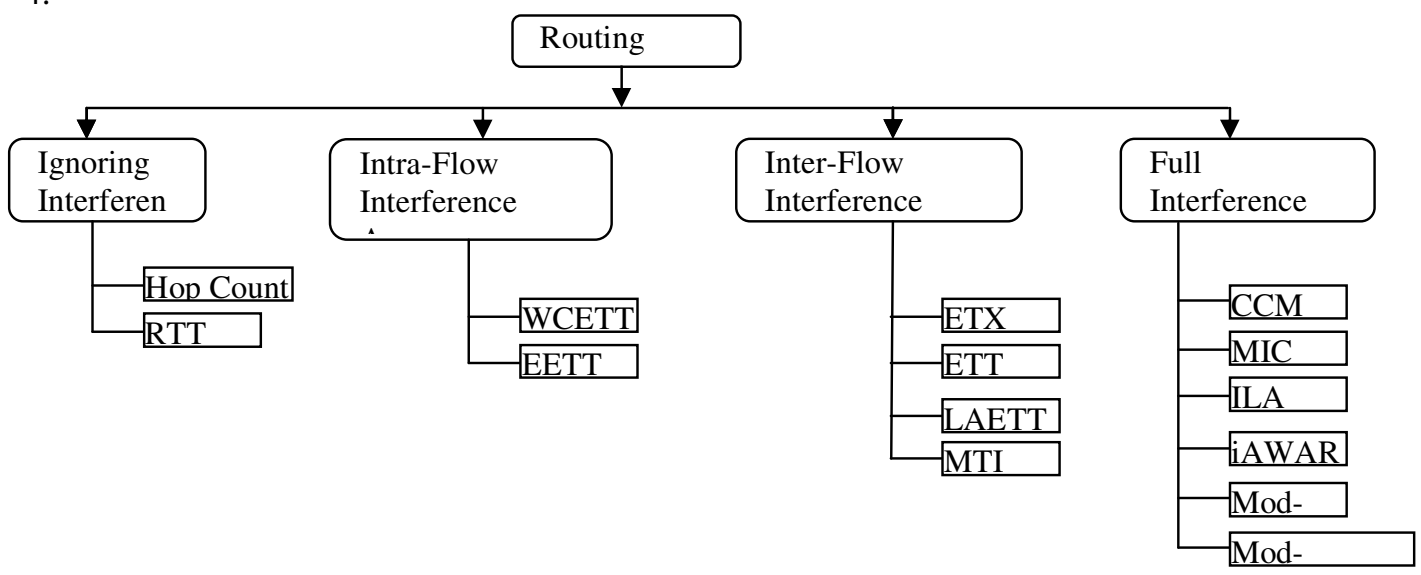

Figure 4. Classification based on Interference

\section{Graph Theoretical Framework For Channel Assignment}

Graph based algorithms have been widely used in many channel assignment algorithms, irrespective of number of radios and channels. The network topology input is generally specified as a connectivity graph. The connectivity graph may be simple undirected graph or multi-graph depending on the number of radios and link topology. This connectivity graph can be converted into an intermediate graph, which generally takes the form of a conflict graph, characterizing the impact of mutual link interferences. For example, when coloring algorithms are used, this conflict graph is fed as input to the graph coloring algorithm which ultimately finds the channel mapping solution for the links. The method is depicted in Figure 5. 


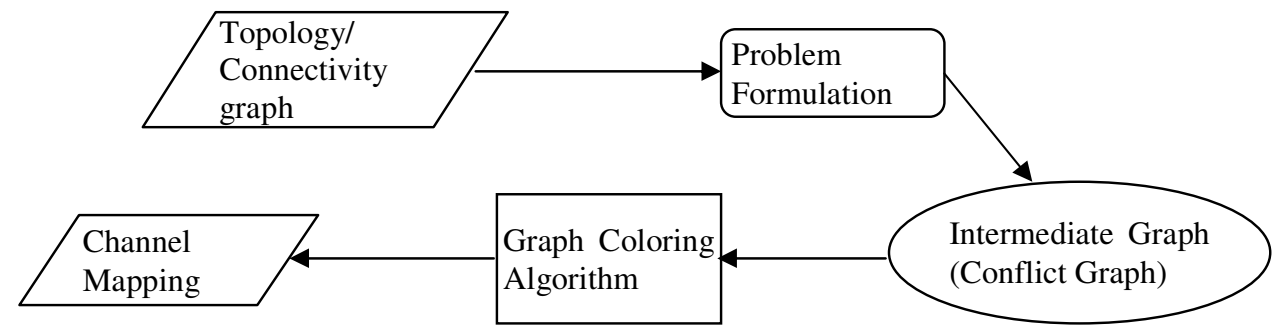

Figure 5. Framework for channel assignment

\subsection{Graphical Representation of Channel Assignment Problems}

Researchers have developed many approaches to design solutions for channel assignment. To formulate the channel assignment problems, different versions of conflict graphs are commonly used to characterize the interference constraints, whereas the application of various graph coloring algorithms has become a popular practice in selecting channels. Below we mentioned some of the graphical models that are very widely used during problem formulation of multi-radio channel assignment:

\subsubsection{Simple Conflict Graph}

A simple conflict graph $\mathrm{G}_{c}\left(\mathrm{~V}_{\mathrm{c}}, \mathrm{E}_{\mathrm{c}}\right)$ is a graph derived from the original network topology graph where each vertex $V_{c}$ represents a communication link or egdeof the topology. There is an edge between two vertices of conflict graph only if the corresponding links in the topology are mutually interfering. An illustration is given in Figure 6. Figure 6(a) shows the original network topology where the three links ij, jp and pqare represented as vertices in Figure 6(b). Here, all the three links interfere with each other because of the close proximity and hence all the three vertices in conflict graph are connected.

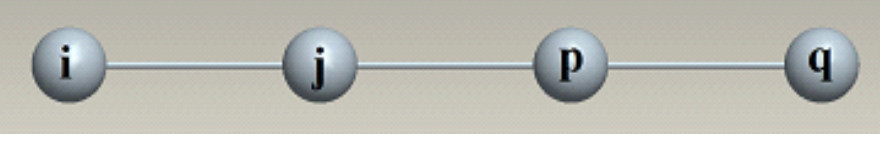

Figure 6(a). A four node network

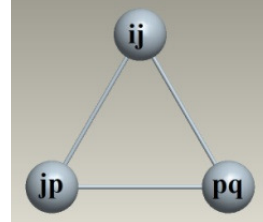

Figure 6(b). corresponding conflict graph

\subsubsection{Weighted Conflict graph}

Some researchers represent the interference effect through assigning various weights to the edges of conflict graph. These types of graphs are known as Weighted Conflict graphs. These weights are assigned based on the extent of interference calculated from appropriate interference model. Two well known algorithms, CLICA [41] and CoSAP [30] are formulated using these models. Of them, the latter is applicable cognitive radio networks.

\subsubsection{Multi-radio Conflict graph}

K. N. Ramachandran et al [12], introduced the notion of Multi-radio Conflict graph (MCG). The authors extended the simple conflict graph to model multi-radio mesh routers (Figure 7). In this model,edges between individual radiosare represented as vertices instead of representing edges between the nodes..Figure 7(a) shows a wireless network with four nodes A,B,C and D where node $\mathrm{C}$ is equipped with 2 radios while the rest have single radio. Figure 
7(b) is the corresponding simple conflict graph while Figure 7(c) shows the multi-radio conflict graph. In the multi-radio conflict graph, all the links connected to node $\mathrm{C}$ are represented with two edges, each for an individual radio.

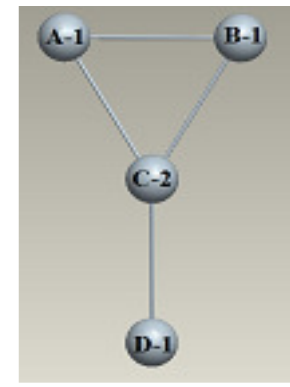

Figure 7(a). A simple | network topology

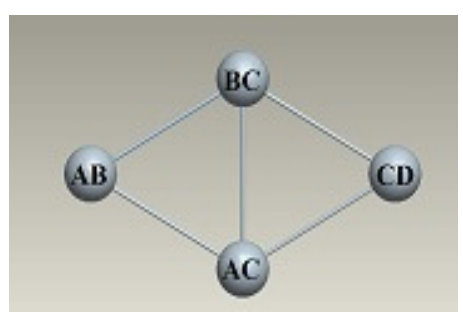

Figure 7(b). Corresponding conflict graph

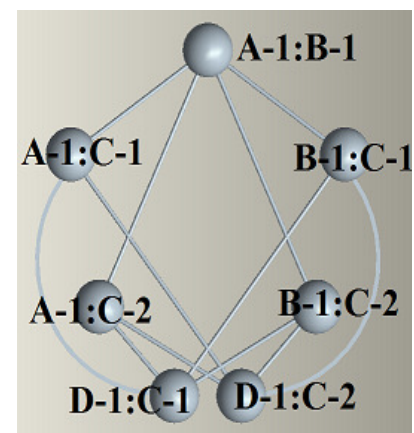

Figure $\quad 7(\mathrm{c})$. Corresponding multiradio conflict graph

\subsubsection{Resource Contention Graph(RCG)}

W. Wang et al. [16] proposed the notion of Resource Contention Graph (RCG)which captures various contention regions in the network topology by identifying all the maximum cliques in the interference graph. The authors described a framework thatrepresents the capacity of a multichannel network when the topology is known. The framework is formulatedas an ILP problem wherethe solution of the problemdetermines the maximum possible spectrumusage for a given topology underchannel and radio constraints. For any specific traffic pattern, the framework provides an upper bound on throughput with optimal routingdecisions. Initially the resource contention graph is generated from thetopology graph. Then a max-flow-likegraph is constructed using the resource contention graph. The Max-flow graph is an extended version of the RCG which is generated by adding a source vertex s and a sink vertex t.For example, Figure8(a) is a topology consisting of 4 nodes. Figure8(b) illustrates the corresponding network flow model. The edge capacity for the first three levels is $\mathrm{N}$, which is the number of channels and the edges of the last two levels have a capacity of $\mathrm{K}$, which is the number of radios.
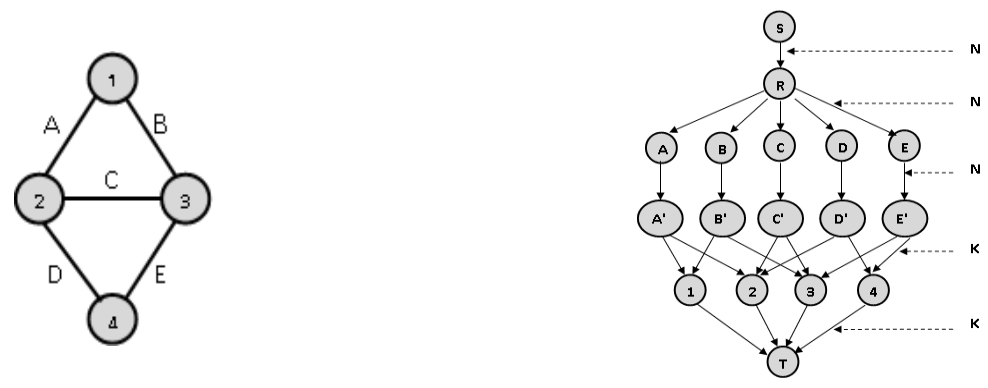

Figure8(a).A topology of 4 nodes Figure8(b).Resource Contention Graph

\subsubsection{Layered Graph Model}

C. Xin et al.[17] proposed a layered graph model to jointly optimize routing and channel assignment. In their model, each layer corresponds toa particular channel. The entire topology is represented using multiple layers of nodes where the number of layer is equal to total 
number of channels. A single network node is shown as a collection of virtual nodes residing in each layer. Vertical edges between layers connect the virtual nodes. The weights of the virtual edges are typicallysetwith a low cost which makes the routing protocol prefer a path with dynamic channel switching. Practically, the cost of the vertical edges should be equal to the cost of channel switching delay. The horizontal edges that belong to the same layer (channel) are the actual cost of air propagation delay. Figure 9 illustrates a simplified layered model of three channels withfour wireless nodes A, B and C, in which A and Dare a communicating pair. The routing path switches from channel 1 to channel 3 at node B and again switches from channel 3 to channel 2 at node $\mathrm{C}$.

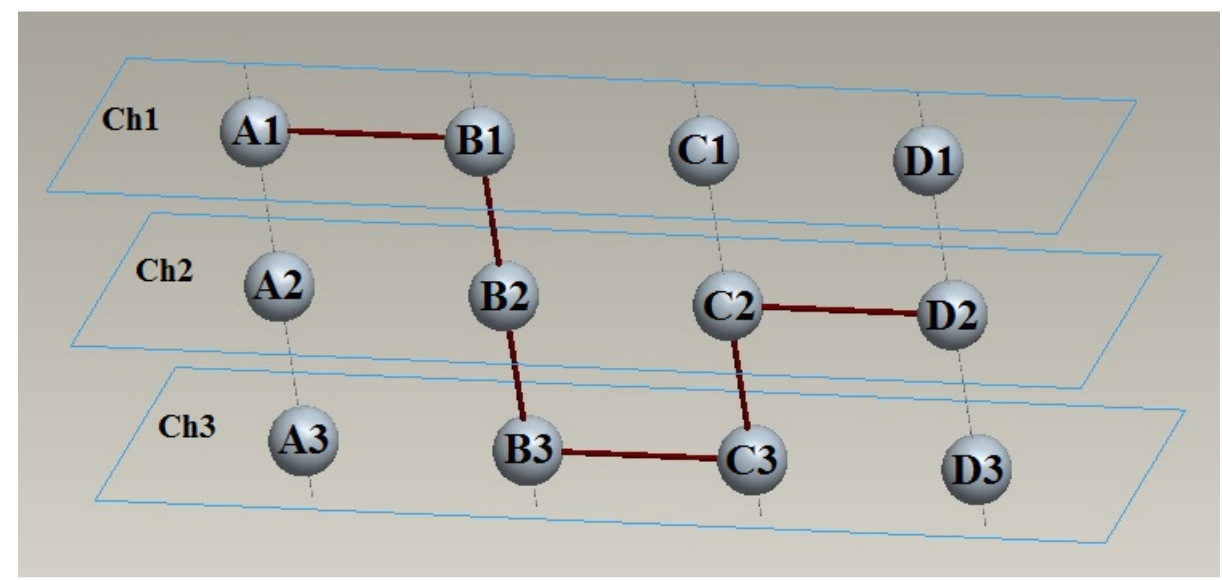

\subsection{Coloring Algorithms}

Figure9. Layered graph Model

Utilizing the different forms of conflict graphs described in the previous section, colors (i.e. channels) have to be assigned to the vertices of the conflict graph (which correspond to the links in the connectivity graph) so that an objective function is optimized. Typical objective functions range from minimizing the difference between the largest and the lowest used colors while avoiding interference to minimizing interferences using a given number of colors. For arbitrary networks, the resulting vertex coloring problems are computationally intractable (i.e., NP-hard). Therefore, the channel assignment problem is usually addressed by means of heuristic approaches, like genetic algorithms, taboo search, saturation degree, simulated annealing etc. Some researchers [52] tend to use polynomial time approximation schemes in greedy approach. Some of the common coloring or partitioning algorithms used to solve the channel assignment problems are Max K-Cut algorithm [32], MIN-MAX k-PARTITION [53], Distance-2 Edge Coloring/Strong Edge Coloring [43] etc.

\section{POC Aware Multi-Radio CA Design Challenges}

\subsection{Improving spectrum utilization through partially overlapped channels (POCs)}

Existing channel assignment algorithms designed for multi-radio multi-channel wireless mesh networks (MRMC-WMN) mainly deal with orthogonal or non-overlapped channels. In fact, due to the adverse effects of adjacent channel interference, almost all channel assignment algorithms use orthogonal channels alone. In reality, the smallnumber of orthogonal channelsposes major challengesin dense networks. For example, the $802.11 \mathrm{~b} / \mathrm{g}$ standards define a total of 11 channels in the US, out of which only 3 areorthogonal (Figure 10). Most 
International Journal of Wireless \& Mobile Networks (IJWMN) Vol. 3, No. 5, October 2011

residential usersand WLAN administrators tune their network interfaces to one of these 3 channels only. Thus two potentially interfering nodes can be assignedto the same channel.This ultimately leads to a wastage of wireless spectrum capacity. Recently it has been revealed that using partially overlapped channels can lead to better utilization of the spectrum. However, an ad-hoc use of POCs can actually degrade performance. Many recent works $[23,28,33,34,56,60,61,62,63,64]$ have shown that the partially overlapped channels (POC) have a great potential for increasing the number of simultaneous transmissions for MRMC-WMN.

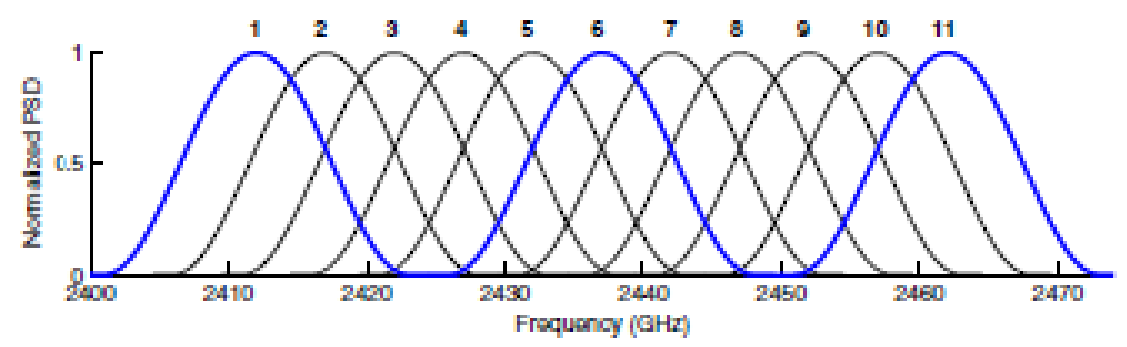

Figure 10. IEEE $802.11 \mathrm{~b} / \mathrm{g}$ channel distribution showing the 3 orthogonal channels in blue [65]

\subsection{Self Interference Problem}

One most critical challenge in POC based channel assignment is to overcome self-interference problem. Links connected to a single node cannot be assigned to channels with overlapping frequency bandwidth due to this problem. To the best of our knowledge, only one of the schemes [23] has identified this problem associated with multi-radio channel assignment. Due to this problem the maximum number of parallel transmission from a single node must be restricted to the number of maximum orthogonal channels available, which is 3 in case of IEEE $802.11 \mathrm{~b} / \mathrm{g}$. Researchers need to concentrate on the severity of this problem and eventually negotiate with this self- interference issue in addition to dealing with Co-Channel interference and ACI. Otherwise, most algorithms would be overestimating the goodness of respective results. For this reason, choosing the appropriate interference model is also very important.

\subsection{Choice of Interference Model}

A fundamental difference between a wireless network and its wired counterpart is that wireless links may interfere with each other, resulting in performance degradation. As a result, there have been numerous researches on wireless networks considering interference between wireless links. Out of several kinds of interference, handling co-channel interference is relatively simpler because many of the wireless link layer protocols use contention resolution mechanisms like RTS-CTS which easily detects if the transmitting channel is busy or not. On the contrary, adjacent channel interferences(ACI) are difficult to detect using channel contention mechanisms because in most cases these ACIs contribute as background noise and reduce the signal to noise ratio. Below, we mention some of the possible choice and alternatives while considering the interference model in a POC based channel assignment algorithm:

Among all the interference models, the Binary Interference Model is the simplest one. The model defines that two links can be either interfering or non-interfering without quantifying the extent of interference among each other. Hence, this phenomenon can be represented as a binary condition. But researchers have proved that this $0 / 1$ assumption in case of interference 
is not true for most cases. The network throughput depends on the actual amount of frequency overlap, distance between nodes and signal to noise ratio which quantifies the interference. Therefore, this model is not meaningful while considering the case of POC based design.

Gupta et. al [58] proposed two important interference model that has been widely used in wireless communication and information theory. These two models, namely protocol model and physical model, have been studied extensively in the literature by subsequent researchers. Inprotocol model, a geographical boundary or interference range is defined for each receiver within which a receiver may perceive interference from other potential transmitters residing inside the boundary where the interfering transmissions are also on the same channel. Hence, this model can capture the effect of co-channel interference but not ACI. On the other hand,in physical model, the interference is mathematically calculated from the signal to noise ratio. Here, a transmission is considered successful when the signal to noise ratioperceived by the receiver exceeds a minimum threshold value after accumulating noise signals contributed byall other transmitters. In this model,the choice of threshold is an important tunable parameter for actual interference measurement. Comparing, protocol and physical model, the latter is obviously the more accurate but the computational complexity is too high. On the other hand, protocol model is easy to calculate but may lead to erroneous results due to inability to capture ACI effect.

A channel interference cost function, proposed by Ko et al. [25], provides a measure of the spectral overlapping level between two partially overlapped channels. The interference cost | between channel $a$ and $b$, denoted by $f(a, b)$, is defined as $f(a, b) \geq 0$ and $f(a, b)=f(b, a)$, where a value of 0 indicates that channels are non-interfering. The value of $f(a, b)$ decreases as the frequency separation between the two channelsincrease. An example of a simple cost | function defined using a single tunable parameter $\delta$ is: $f(a, b)=\max (0, \delta-|a-b|)$ where $\delta$ can be defined as the minimum channel separation between two non-overlapping channels. For IEEE $802.11 \mathrm{~b} / \mathrm{g}, \delta=5$. For example, if $a=7$ and $b=4$, then $f(7,4)=\max (0,5-3)=2$. Again, for $a=9$ and $b=2, f(9,2)=\max (0,5-3)$, which means no interference at all. Due to the simplicity of this cost function, it is also easy to implement in a channel assignment algorithm as a measure of partial interference.

Interference-factor or simply I-factor,proposed by the current authors [57], can also be regarded a suitable measure of the extent of channel overlap. If $P_{i}$ denotes the power received at a given location of a particular signal on channel $i$, and $P_{j}$ denote the power received of the same signal at the same location on channel $\mathrm{j}$, then the interference factor between $i$ and $j, I(i, j)$ is defined as $\frac{P_{i}}{P_{j}} . I(i, j)$ gives the fraction of a signal's power on channel $\mathrm{j}$ that will be received on channel i. I-factor can be calculated analytically as well as empirically and does not depend on the radio propagation properties of the environment (i.e. open space or indoors). It depends on the extent of frequency overlap between the signals on channels $i$ and $j$. Hence, this is a suitable choice for POC based interference models. For example, some POC based channel assignment schemes have been proposed [56, 57, 63] using this interference model. Table III below shows I $(\mathrm{i} ; 6)$ normalized to a scale of $0 \ldots 1$.

Table 3.I $(i, 6)$ Values [56]

\begin{tabular}{|c|c|c|c|c|c|c|c|c|c|c|c|}
\hline Channel & 1 & 2 & 3 & 4 & 5 & 6 & 7 & 8 & 9 & 10 & 11 \\
\hline Normalized SNR (I-factor) & 0 & 0.22 & 0.60 & 0.72 & 0.77 & 1.0 & 0.96 & 0.77 & 0.66 & 0.39 & 0 \\
\hline
\end{tabular}

An innovative Channel Overlapping Matrix Model has been introduced by A. Hamed Rad et. al [28]. The model captures the interference using a channel overlapping matrix. For example, 
let us consider an MRMC-WMN where $\mathrm{N}$ denotes the set of wireless routers whereeach router is equipped with I NICs. There are a total of $\mathrm{C}$ channels available for transmission. For any two routers $a, b \in N, \mathrm{a} C \times 1$ channel assignment vector is defined $\overline{x_{a b}}$.If router $a$, communicates with router $\mathrm{b}$ over the $i$ th channel, then the $i$ th element in $\overline{x_{a b}}$ is equal to 1 ; otherwise, it is equal to zero. As for example, a router $a$ islinked with router $b$ through the 2 nd | channel where $\mathrm{C}=5$. This implies, $\overline{x_{a b}}=\left[\begin{array}{lllll}0 & 1 & 0 & 0 & 0\end{array}\right]^{T}$.Let, $\mathrm{m}$ and $\mathrm{n}$ are two of the available channels within the frequency band. To mathematically model the overlapping effect among different channels, the authors defined a symmetric $C \times C$ channel overlapping matrix $\mathrm{W}$.

| The entry in the $m$-th row and the $n$-th column of $\mathrm{W}$ is denoted by scalar $w_{m n}$ and is defined to be as follows:

$$
w_{m n}=\frac{\int_{-\infty}^{\infty} F_{m}(\omega) F_{n}(\omega) d \omega}{\int_{-\infty}^{\infty} F_{m}^{2}(\omega) d \omega}
$$

Where $F_{m}(\omega)$ and $F_{n}(\omega)$ denote the respective power spectral densities on channels $\mathrm{m}$ and $\mathrm{n}$.

\section{Future Research Directions}

\subsection{Multi-Radio Multi-Channel Concerns}

Despite significant amount of research [16], the network capacity of WMNs is still a challenging topic. Although Vaidyaet. al. [22, 43, 44] characterized network capacity in terms of number of channels and radios as well as switching delay, more conditions can be added such as heterogeneous radios, mobility of nodes. On the other hand, Wang et. al. [16] proposed a framework to maximize overall capacity based on graph theoretical approach.In addition, as of to-date, no MRMC protocol exploits the multi-rate capability of current 802.11 wireless cards. By considering only homogeneous links, the problem becomes much simpler. However, a protocol with adaptive rates can achieve better performance.

The channel switching delay is an important concern for channel assignment schemes that switch the radio interfaces very frequently. Despite of significant improvement in wireless networking hardware, channel switching delay is still in the order of millisecond which is considered as an overhead for overall end-to-end delay. On the other hand, using a static channel assignment approach to avail the benefits of reduced overhead and stable topology will lack from the capacity improvement gained by MRMC environment. Therefore, a well estimated tradeoff is necessary to overcome the problem arising from switching overhead.

\subsection{POC Aware Design}

The wireless literature still lacks an efficientPOC based dynamic and distributed algorithm, a algorithm that can handle channel switching for each node. Though some static schemes have been designed with POC [56, 60, 61, 62, 63, 64], more emphasis should be on dynamic versions.No existing simulator is capable of simulating such MRMC networks that involve interference calculated from POCs. Hence current popular simulators might be extended with features supporting POC channel model and network protocolsdesigned for partially overlapped channels. As of this date, there is no joint routing and channel assignment algorithm designed with POCs. Polynomial time approximation schemes are often considered as feasible solution in this area where many critical factors, such as compound routing metrics that characterizes appropriate interference model, should be handled. 
International Journal of Wireless \& Mobile Networks (IJWMN) Vol. 3, No. 5, October 2011

Further, tuning the interference tolerance level by carefully adjusting the SINR threshold value is of great importance. A higher threshold value will definitely give better transmission quality with low interferences and noises, but will have higher probability of retransmission and low throughput, whereas, a small threshold will generate higher interference and degrade the quality of signal reception. Thus a tradeoff has to be made in case of deciding the SINR threshold value.

\section{CONCLUSION}

In this paper we have identified the key challenges and research approaches associated with assigning channels to radio interfaces in multi-radio wireless mesh networks. We have provided the goals and objectives of an efficient algorithm, classification of existing schemes and comparative analysis of different schemes. We presented the challenges involved with multi-radio and POC based design. In the end, we outlined important open research issues for future investigations.

\section{REFERENCES}

[1] E. Hossain, Kin KwongLeung (2008) Wireless Mesh Networks: Architectures And Protocols, Springer

[2] P. Kyasanur and N.H. Vaidya (2006) Routing and Link-layer Protocols for Multi-Channel MultiInterface Ad Hoc Wireless Networks. In MobiCom.

[3] J.Crichigno,M. Wu, W.Shu (2008) Protocols and architectures for channel assignment inwireless mesh networks, Ad hoc Networks, Vol-6.

[4] D. S. J. D. Couto, D. Aguayo, J. Bicket, and R. Morris (2003) A high-throughput path metric for multi-hop wireless routing. In MobiCom.

[5] R.Draves, J. Padhye, and B. Zill (2004) Routing in multi-radio, multihop wireless mesh networks. In MobiCom.

[6] J. So, N.H. Vaidya (2004) Multi-Channel MAC for Ad Hoc Networks: Handling Multi-Channel Hidden Terminals Using A Single Transceiver. In MobiHoc.

[7] P. Jacquet, P. Muhletaler, T. Clausen, A. Laouiti, A. Qayyum, and L. Viennot (2001) Optimized Link State Routing Protocol for Ad Hoc Networks. In IEEE INMIC.

[8] D. B. Johnson and D. A. Maltz (1996) Dynamic source routing in ad hoc wireless networks. In Mobile Computing, Vol-353.

[9] X. Lin and S. Rasool (2007) A distributed joint channel-assignment, scheduling and routing algorithm for multi-channel ad hoc wireless networks. In IEEE INFOCOM'07.

[10] J. C. Park and S. K. Kasera (2005) Expected Data Rate: An Accurate High-Throughput Path Metric For Multi-Hop Wireless Routing. In IEEE SECON.

[11] C. E. Perkins, E. M. Royer, and S. Das (2003) Ad-hoc On Demand Distance Vector (AODV) Routing. http://www.ietf.org/rfc/ rfc3561.txt.

[12] K. N. Ramachandran, E. M. Belding, K. C. Almeroth, and M. M. Buddhikot (2006) Interferenceaware channel assignment in multi-radio wireless mesh networks. In Infocom.

[13] A. Raniwala and T. Chiueh (2005) Architecture and algorithms for an ieee 802.11-based multichannel wireless mesh network. In IEEE Infocom.

[14] A. Raniwala, K. Gopalan, and T. ckerChiueh (2004) Centralized channel assignment and routing algorithms for multi-channel wireless mesh networks. SIGMOBILE Mob.Comput.Commun.Rev. Vol-8.

[15]PradeepKyasanur and NitinVaidya (2005) "Multi-Channel Wireless Networks: Capacity and Protocols," Technical Report, University of Illinois at Urbana-Champaign. 
International Journal of Wireless \& Mobile Networks (IJWMN) Vol. 3, No. 5, October 2011

[16] W. Wang and X. Liu (2006) A Framework for Maximum Capacity in Multi-channel Multi-radio Wireless Networks.In IEEE CCNC.

[17] C. Xin and C.-C.Shen (2005)A Novel Layered Graph Model for Topology Formation and Routing in Dynamic Spectrum Access Networks. In IEEE DySPAN.

[18] Y. Yang, J. Wang, and R. Kravets (2005) Interference-aware Load Balancing for Multihop Wireless Networks. In Tech Report UIUCDCS-R-2005-2526, Department of Computer Science, UIUC.

[19] J. So, N.H. Vaidya (2004) A Routing Protocol for Utilizing Multiple channels in Multi-hop Wireless Networks with a Single Transceiver, UIUC Technical Report.

[20] R. Vedantham, S. Kakumanu, S. Lakshmanan and R. Sivakumar (2006) "Component Based Channel Assignment in Single Radio, Multi-channel Ad Hoc Networks," InMobiCom.

[21]Weirong Jiang, ShupingLiu, Yun Zhu and Zhiming Zhang (2007) Optimizing Routing Metrics for Large-Scale Multi-Radio Mesh Network, In WiCom.

[22] P. Kyasanur, N. Vaidya (2005) Capacity of multi-channel wireless networks: Impact of number of channels and interfaces, In MobiCom.

[23] ZhenhuaFeng, Yaling Yang (2008) How Much Improvement Can We Get From Partially Overlapped Channels? , In IEEE WCNC.

[24] Xiaoguang Li, ChangqiaoXu (2009) Joint Channel Assignment and Routing in Real Time Wireless Mesh Network, In IEEE WCNC.

[25] Bong-Jun Ko Vishal, MisraJitendra, Padhye Dan Rubenstein (2007)Distributed Channel Assignment in Multi-Radio 802.11 Mesh Networks, In IEEE WCNC.

[26] Ka-Hung Hui, Wing-Cheong Lau, On-ChingYue (2007) Characterizing and Exploiting Partial Interference in Wireless Mesh Networks, In IEEE ICC.

[27] MansoorAlicherry,Randeep Bhatia, Li (Erran) Li (2005) Joint Channel Assignment and Routing for Throughput Optimization in Multiradio Wireless Mesh Networks, In MobiCom.

[28] A. HamedMohsenian Rad, Vincent W.S Wong (2007) Partially Overlapped Channel Assignment for Multi-Channel Wireless Mesh Networks, In IEEE ICC.

[29] Hua Yu, PrasantMohapatra, Xin Liu (2008) Channel assignment and link scheduling in multi-radio multi-channel wireless mesh networks, ACM Mobile Networks and Applications, Vol. 13.

[30] Anthony Plummer Jr., TaoWu, SubirBiswas (2007) A Cognitive Spectrum Assignment Protocol using Distributed Conflict Graph Construction, In IEEE MILCOM.

[31] Seongho CHO, Chong-kwon KIM (2008) Interference-Aware Multi- Channel Assignment in Multi-Radio Wireless Mesh Networks, IEICE Transactions on Communications Vol. E91-B(5).

[32] AnandPrabhu Subramanian, Himanshu Gupta, Samir R. Das (2008) Minimum Interference Channel Assignment in Multi-Radio Wireless Mesh Networks, IEEE Transactions on Mobile Computing, Vol. 7.

[33] Arunesh Mishra, Eric Rozner, Suman Banerjee, William Arbaugh (2005) Exploiting Partially Overlapping Channels inWireless Networks: Turning a Peril into an Advantage, Internet Measurement Conference.

[34] Arunesh Mishra, VivekShrivastava, Suman Banerjee, William Arbaugh (2006) Partially Overlapped Channels Not Considered Harmful, ACM SIGMETRICS Vol. 34.

[35] Vijay Raman, Nitin H. Vaidya (2009) Adjacent Channel Interference Reduction in Multichannel Wireless Networks Using Intelligent Channel Allocation, Technical Report, ICWS, UIUC.

[36] Stefano Avallone, Ian F. Akyildiz (2008) A channel assignment algorithm for multi-radio wireless mesh networks, Computer Communications, Vol. 31.

[37] Haitao Wu et. al (2006) Distributed Channel Assignment and Routing in Multiradio Multichannel Multihop Wireless Networks, IEEE Journal on Selected Areas in Communications, Vol. 24. 
International Journal of Wireless \& Mobile Networks (IJWMN) Vol. 3, No. 5, October 2011

[38] VartikaBhandaria, NitinVaidya (2008) Heterogeneous Multi-Channel Wireless Networks: Routing and Link Layer Protocols, Mobile Computing and Communications Review, Vol.12.

[39] Ian F. Akyildiz, X. Wang (2005) A Survey on Wireless Mesh Networks, IEEE Radio Communications.

[40] HabibaSkalli, SamikGhosh and Sajal K. Das, Luciano Lenzini, Marco Conti (2007) Channel Assignment Strategies for Multiradio Wireless Mesh Networks: Issues and Solutions, IEEE Communications Magazine.

[41] M. Marina and S. R. Das (2005) "A Topology Control Approach for Utilizing Multiple Channels in Multi-Radio Wireless Mesh Networks," In Proc. Broadnets.

[42] V. Bhandari and N. H. Vaidya (2007) Heterogeneous multi-channel wireless networks: Scheduling and routing issues. Technical Report, UIUC.

[43] V. Bhandari and N. H. Vaidya (2007) Capacity of multichannel wireless networks with random (c, f) assignment.In MobiHoc.

[44] V. Bhandari and N. H. Vaidya (2007) Connectivity and Capacity of Multichannel Wireless Networks with Channel Switching Constraints.In IEEE INFOCOM.

[45] Y. Yang, J. Wang, R. Kravets (2005) Designing routing metrics for mesh networks, In IEEE WiMesh.

[46] C.L. Barrett, G.Istrate, V. S. A.Kumar, M.V. Marathe, S.Thite, S.Thulasidasan (2006) Strong Edge Coloring for Channel Assignment in Wireless Radio Networks, In IEEEPerCom.

[47] Bharat Kumar Addagada,VineethKisara and Kiran Desai (2009)A Survey: Routing Metrics for Wireless Mesh Networks.

[48] DevuManikantanShila and Tricha Anjali (2008) Load-aware Traffic Engineering for Mesh Networks, Computer Communications, Vol-31.

[49] HervéAïache, Laure Lebrun, Vania Conan and Stéphane Rousseau (2008) LAETT A load dependent metricfor balancing Internet tra-c in Wireless Mesh Networks, In IEEE MASS.

[50] AnandPrabhuSubramanian ,Milind M. Buddhikot and Scott Miller (2006) Interference Aware Routing in Multi-Radio Wireless Mesh Networks.In IEEEWiMesh.

[51] Weirong Jiang, Shuping Liu, Yun Zhu and Zhiming Zhang (2007) Optimizing Routing Metrics for Large-Scale Multi-Radio Mesh Networks. In WiCOM

[52] SartajSahni and Teofilo Gonzalez (1976) NP-complete approximation problems, Journal of the Association for Computing Machinery, Vol. 23.

[53] Das, A.K.; Vijayakumar, R.; Roy, S (2006) WLC30-4: Static Channel Assignment in Multi-radio Multi-Channel 802.11 Wireless Mesh Networks: Issues, Metrics and Algorithms,In IEEE Globecom.

[54] PradeepKyasanur, ChandrakanthChereddi, Nitin H. Vaidya (2006) Net-X: System eXtensions for Supporting Multiple Channels, Multiple Interfaces, and Other Interface Capabilities. UIUC Technical Report.

[55] Xiaoyan Hong, BoGu, Mohammad Hoque, Lei Tang (2010) Exploring multiple radios and multiple channels in wireless mesh networks, IEEE Wireless Communications, Vol. 17.

[56] Mohammad Hoque, Xiaoyan Hong, FarhanaAfroz (2009)Multiple Radio Channel Assignment Utilizing Partially Overlapped Channels, In IEEEGlobecom.

[57] Mohammad A Hoque, Xiaoyan Hong, (2009)Interference Minimizing Channel Assignment Using Partially Overlapped Channels in Multi-radio Multi-channel Wireless Mesh Networks, IEEE Southeast Conference.

[58] P.Guptaand P. R. Kumar(2000)The Capacity of Wireless Networks, IEEE Trans. on Information Theory, Vol. 46, No. 2.

[59] Mohammad A. Hoque, Xiaoyan Hong, Md. Ashfakul Islam, KaziZunnurhain (2010) "Delay analysis of Wireless Ad Hoc networks: Single vs. multiple radio," In Proceedings of IEEE LCN. 
International Journal of Wireless \& Mobile Networks (IJWMN) Vol. 3, No. 5, October 2011

[60] K. Shih, C. Chang, D. Deng, and H. Chen (2010) Improving Channel Utilization by Exploiting Partially OverlappingChannels in Wireless Ad Hoc Networks,In IEEE Globecom.

[61] Yuting Liu, R. Venkatesan, and Cheng Li (2010) Load-Aware Channel Assignment Exploiting Partially Overlapping Channels for Wireless Mesh Networks,In IEEE Globecom.

[62] Yuting Liu, R. Venkatesan, and Cheng Li (2009) Channel assignment exploiting partially overlapping channels for wireless mesh networks, In IEEE Globecom.

[63] Pedro B. F. Duarte, Zubair Md. Fadlullah, Kazuo Hashimoto, and Nei Kato (2010) Partially Overlapped Channel Assignment on Wireless Mesh Network Backbone, In IEEE Globecom.

[64] Abbasi, S.,Kalhoro, Q., Kalhoro, M.A (2011) Efficient Use of Partially Overlapped Channels in 2.4 GHz WLAN Backhaul Links, International Conference on Innovations in Information Technology.

[65] http://www.cisco.com/en/US/docs/solutions/Enterprise/Mobility/emob30dg/RFDesign.html.

\section{Authors}

MOHAMMAD ASADUL HOQUE is a Ph.D. student in the Department of Computer Science at the University of Alabama. He completed his M.S. in computer science from the same university in 2010. After completing his B.S. in Computer Science and Engineering from Bangladesh University of Engineering and Technology in 2006, he joined the industry as a network engineer. His current research interest is in the area of wireless ad hoc networks and cognitive radio networks.

XIAOYAN HONG is an associate professor in the Department of Computer Science at the University of Alabama. She received her Ph.D. degree in computer science from the University of California, Los Angeles in 2003. Her research interests include mobile and wireless networks, challenged networks, and the future Internet. Her current research focuses on mobility, routing, bio-inspired communications, and wireless network trust and privacy.
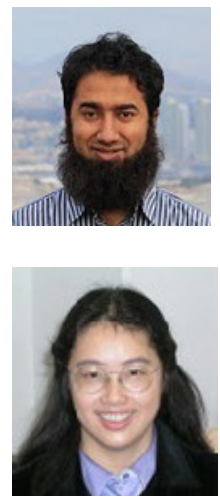